\title{
Applications of the Differential Transformation Method and Multi-Step Differential Transformation Method to Solve a Rotavirus Epidemic Model
}

\author{
Pakwan Riyapan $^{1, *}$, Sherif Eneye Shuaib ${ }^{1}$, Arthit Intarasit ${ }^{1}$, Khanchit Chuarkham ${ }^{2}$ \\ ${ }^{1}$ Department of Mathematics and Computer Science, Faculty of Science and Technology, Prince of Songkla University, Pattani, 94000, Thailand \\ ${ }^{2}$ Faculty of Commerce and Management, Prince of Songkla University, Trang Campus, Trang 92000, Thailand
}

Received December 23, 2020; Revised February 12, 2021; Accepted February 23, 2021

Cite This Paper in the following Citation Styles

(a): [1] Pakwan Riyapan, Sherif Eneye Shuaib, Arthit Intarasit, Khanchit Chuarkham, "Applications of the Differential Transformation Method and Multi-Step Differential Transformation Method to Solve a Rotavirus Epidemic Model," Mathematics and Statistics, Vol. 9, No. 1, pp. 71-80, 2021. DOI: 10.13189/ms.2021.090112.

(b): Pakwan Riyapan, Sherif Eneye Shuaib, Arthit Intarasit, Khanchit Chuarkham, (2021). Applications of the Differential Transformation Method and Multi-Step Differential Transformation Method to Solve a Rotavirus Epidemic Model. Mathematics and Statistics, 9(1), 71-80. DOI: 10.13189/ms.2021.090112.

Copyright @2021 by authors, all rights reserved. Authors agree that this article remains permanently open access under the terms of the Creative Commons Attribution License 4.0 International License

\begin{abstract}
Epidemic models are essential in understanding the transmission dynamics of diseases. These models are often formulated using differential equations. A variety of methods, which includes approximate, exact and purely numerical, are often used to find the solutions of the differential equations. However, most of these methods are computationally intensive or require symbolic computations. This article presents the Differential Transformation Method (DTM) and Multi-Step Differential Transformation Method (MSDTM) to find the approximate series solutions of an SVIR rotavirus epidemic model. The SVIR model is formulated using the nonlinear first-order ordinary differential equations, where $S, V, I$ and $R$ are the susceptible, vaccinated, infected and recovered compartments. We begin by discussing the theoretical background and the mathematical operations of the DTM and MSDTM. Next, the DTM and MSDTM are applied to compute the solutions of the SVIR rotavirus epidemic model. Lastly, to investigate the efficiency and reliability of both methods, solutions obtained from the DTM and MSDTM are compared with the solutions from the Runge-Kutta Order 4 (RK4) method. The solutions from the DTM and MSDTM are in good agreement with the solutions from the RK4 method. However, the comparison results show that the MSDTM is more efficient and converges to the RK4 method than the DTM. The advantage of the DTM and MSDTM over other methods is that it does not require a perturbation parameter to work and does not generate secular terms. Therefore the application of both methods can be extended to other compartmental models.
\end{abstract}

Keywords Differential Transformation Method, Multi-step Differential Transformation Method, Rotavirus

\section{Introduction}

Epidemic diseases remain an increasing threat worldwide challenging researchers, decision-makers, and public health officers to monitor, identify, and respond to near-real-time infection outbreaks [1]. Mathematical models are essential toolkits to understand epidemic diseases because they aid scientists in analyzing and predicting disease mechanisms and their future outbreaks. The fundamental procedure in modelling the spread of disease is through the use of compartmental models.

Compartmental models are described using a set of mathematical equations to understand how individuals in various "compartments" in a population interact with one another [2]. The most common compartmental models are deterministic models which can be divided into ordinary differential equations (ODEs) or partial differential equations (PDE). The SIR model is an example of a compartment model widely used to study epidemic diseases. This model describes the movement of human between the susceptible $S$, infective $I$ and removed $R$ compartments, respectively. There are several modifications to the SIR model, such as SIS, SEIS, SEIR, and SVIR models. The SIS model describes the movement of humans from the susceptible compartment to the infective compartment and 
back to the susceptible compartment.

On the other hand, the SEIS and SEIR models comprise of an exposed period from being infected to become infective [3]. Furthermore, the SIR model can be modified to model with vaccination. This can be achieved by introducing an additional compartment, $V$, to the SIR model, for vaccinated individuals.

The simple formation of mathematical models is not sufficient enough to control epidemic disease spread because most of the biological problems formulated in model frameworks are often nonlinear and challenging to solve. To compute the solutions of these models, scientists often employ approximate or perturbation methods [4]. Examples of such methods are the Adomian Decomposition Method [5], Homotopy Analysis Method [6], and the Homotopy Perturbation Method [7, 8]. However, due to the rise in large scale international human movement, human and animal population displacements, and the complexity of modern society, more effective methods are required to solve these models.

The Differential Transformation Method (DTM) is a numerical-analytical approach derived from Taylor's series expansion. In the DTM, a given system of equations with initial conditions is reconstructed into a system of recurrence equations that results in a system of an algebraic equation. The Taylor series method usually requires large computational work for finding the higher-order derivation of a function. The main advantage of the DTM is its direct application to linear and nonlinear ODEs without discretization, linearization, or perturbation. Another advantage is that it can reduce the size of computations to be carried out and, at the same time, provide a fast convergence rate and accurate series solution. However, its drawback is that a truncated series solution is often obtained after solving the differential equation. Though the truncated solution does not show the system's exact behaviors, it gives an excellent approximation when a small region is considered. Some recent applications of the DTM to epidemiological models include the studies of Ndii et al. (2018), Peter et al. (2018), Kharrat and Toma (2019) and Christopher et al. (2020) [9, 10, 11, 12]. Ndii et al. (2018) [9] applied the DTM to compute the analytical and numerical solutions of a dengue transmission mathematical model and compared the results with the RK4 method. Peter et al. (2018) [10] employed the DTM to solve a typhoid fever model for a given constant population and compare the obtained solutions from the DTM with the RK4 method. Kharrat and Toma (2019) [11] the DTM to linear and nonlinear initial and boundary value problems are given in the form of ordinary differential equations of 14th order. In their study, the capability and robustness of the DTM were proved by solving examples and then compared the results of the numerical examples with the Homotopy perturbation method (HPM) and the exact solutions. Christopher et al. (2020) [12] used the DTM to carry out a dynamic analysis of the COVID-19 epidemic model.

To overcome the drawback of the DTM mentioned earlier, the Multi-Step Differential Transformation method (MSDTM) presented in the studies of $[13,14,15]$ have been used. In MSDTM, the interval $[0, T]$ is divided into $M$ sub-intervals, and the series solutions are obtained in $t \in\left[t_{i}, t_{i+1}\right], \quad i=$ $1,2, \ldots, M-1$. The application of the MSDTM to epidemic models can be found in the studies of $[16,17]$. Gökdoğan et al. (2012) [16] applied the MSDTM to find the approximate solution of the Hantavirus infection model. Also, Astuti et al. (2019) [17] employed MSDTM to solve an Influenza virus model. In the study of Gökdoğan et al. (2012) the SI model was used while the SEIR model was employed in the study of Astuti et al. (2019).

Since vaccination is the most effective means to reduce the spread of rotavirus infection, the solutions of a model framework designed to predict the optimal coverage level of rotavirus vaccines are necessary. A thorough search of relevant works of literature on the solution of the SVIR model using MSDTM yielded no results. Thus, this study will seek to extend the application of the MSDTM to solve an SVIR rotavirus epidemic model proposed in the study of Omondi et al. (2015) [18].

This paper is organized as follows: in the "Materials and Methods" section, we present the rotavirus epidemic model, the description of DTM and MSDTM and the applications of the DTM and MSDTM to solve the SVIR model, respectively. Then, in the "Result and Discussion" section, the numerical experiments carried out with the model, the graphical plot, and error difference between the MSDTM and RK4 are presented. Finally, the conclusions from this study are given in the "Conclusions" section.

\section{Materials and Methods}

\subsection{Rotavirus Epidemic Model}

Rotavirus diarrhea is a common childhood epidemic disease. Infants and children below the age of five are most susceptible to rotavirus diarrhea. The transmission of rotavirus infection is mainly through person to person, respiratory droplets, contamination of objects, hands, water, and food with infected feces. There is presently no cure for rotavirus infection, and vaccination also remains the most effective method to reduce the spread of rotavirus.

Several mathematical models incorporating vaccination have been formulated to understand the dynamics of rotavirus epidemics. In this study, we consider the SVIR model proposed in the study of Omondi et al. (2015). Equation (1) denotes the SVIR model:

$$
\begin{aligned}
\frac{d S}{d t} & =(1-\theta) \lambda-\beta S I+\zeta V-\eta S-\mu S, \\
\frac{d V}{d t} & =\theta \lambda+\eta S-\xi \beta V I-(\zeta+\mu) V, \\
\frac{d I}{d t} & =\beta S I+\xi \beta V I+(\tau+\varpi+\mu) I, \\
\frac{d R}{d t} & =\varpi I-\mu R,
\end{aligned}
$$

with non-negative initial conditions.

In (1), the human recruitment rate to the susceptible compartment is denoted as $(1-\theta) \lambda$. The recruitment rate of individuals to the vaccinated compartment is given as $\theta \lambda . \eta$ means the rate at which children in the susceptible class are vaccinated while $\zeta$ represents the waning rate of the vaccine. The effective 
contact rate for disease transmission to occur is denoted as $\beta$. The parameter $0<\xi<1$ represents the expected reduction in infection risk due to vaccination. The death rate due to rotavirus disease takes place at the rate $\tau$ while $\varpi$ denotes the rate at which children move to the recovered class. The parameter $\mu$ denotes the natural death rate of the population.

\subsection{Description of DTM}

To gain a proper understanding of the DTM, some basic definitions of DTM adopted from the study of Hassan (2004) [19] are given below:

Definition 1. The differential transformation $P(k)$ of a function $p(t)$ is defined as follows:

$$
P(k)=\frac{1}{k !}\left[\frac{d^{k} p(t)}{d x^{k}}\right]_{x=0},
$$

where $p(t)$ is the original function and $P(k)$ is the transformed function.

Definition 2. The differential inverse transformation of $P(k)$ is defined as follows:

$$
p(t)=\sum_{k=0}^{\infty} x^{k} P(k) .
$$

From (2) and (3), respectively, the function $p(t)$ expanded in Taylor series around a point $x=0$ is defined as follows:

$$
p(t)=\sum_{k=0}^{\infty} \frac{x^{k}}{k !}\left[\frac{d^{k} p(t)}{d x^{k}}\right]_{x=0} .
$$

There are several basic mathematical operations relating to DTM. However, in this study, we will only list the operations used in deriving solutions of the SVIR model. Table 1 contains the list of mathematical operations of the DTM as referenced from the study of Batiha and Batiha (2011) [20]:

\subsection{Description of MSDTM}

For an interval $[0, T]$, the approximate solution of an initial value problem obtained by using DTM can be expressed as infinite series given in (5)

$$
p(t)=\sum_{k=0}^{\infty} P(k) t^{k}, t \in[0, T] .
$$

Assuming the interval $[0, T]$ is divided into $M$ subintervals $\left[t_{i-1}, t_{i}\right], \quad i=1,2, \ldots, M$ of equal step size $h=T / M$, the steps to solve an ordinary differential equation using the MSDTM can be described as follows: firstly, the DTM is applied to the given ODE over the interval $\left[0, t_{1}\right]$. To do this, we obtain

$$
p_{1}(t)=\sum_{k=0}^{N} P_{1}(k) t^{k}, t \in\left[0, t_{1}\right] .
$$

For the next time step, the value at $t=t_{1}$ is used as an initial condition. The initial condition for the next time step is given as

$$
p_{m}\left(t_{m-1}\right)=p_{m-1}\left(t_{m-1}\right) .
$$

The process is repeated to generate a sequence of approximate solutions $p_{m}(t)=1,2, \ldots, M$ for the solution of $p(t)$

$$
p_{m}(t)=\sum_{k=0}^{N} P_{m}(k)\left(t-t_{m-1}\right)^{k}, t \in\left[t_{m-1}, t_{m}\right] .
$$

Let $m=M$, then the MSDTM gives the following solutions below:

$$
\begin{aligned}
p_{1}(t)= & \sum_{k=0}^{N} P_{1}(k)\left(t-t_{0}\right)^{k} \\
= & P_{1}(0)+P_{1}(1)\left(t-t_{0}\right)+P_{1}(2)\left(t-t_{0}\right)^{2} \\
& +\cdots+P_{1}(N)\left(t-t_{0}\right)^{N}, t \in\left[0, t_{1}\right], \\
p_{2}(t)= & \sum_{k=0}^{N} P_{2}(k)\left(t-t_{1}\right)^{k} \\
= & P_{2}(0)+P_{2}(1)\left(t-t_{1}\right)+P_{2}(2)\left(t-t_{1}\right)^{2} \\
& +\cdots+P_{2}(N)\left(t-t_{1}\right)^{N}, t \in\left[t_{1}, t_{2}\right], \\
& \vdots \\
p_{M}(t)= & \sum_{k=0}^{N} P_{M}(k)\left(t-t_{M-1}\right)^{k} \\
= & P_{M}(0)+P_{M}(1)\left(t-t_{M-1}\right)+P_{M}(2)\left(t-t_{M-1}\right)^{2} \\
& +\cdots+P_{M}(N)\left(t-t_{M-1}\right)^{N}, t \in\left[t_{M-1}, t_{M}\right] . \quad(8)
\end{aligned}
$$

The above equations are the MSDTM solutions for each time step.

\subsection{Application of the DTM and MSDTM to the SVIR Rotavirus Model}

By using the mathematical operations given in Table 1, the recurrence relation obtained to the system in (1) using DTM is given below:

$$
\begin{aligned}
S(k+1) & =\frac{1}{k+1}((\lambda-\lambda \theta) \delta(k)+\zeta V(k)-\eta S(k)-\mu S(k)) \\
& -\frac{1}{k+1}\left(\beta \sum_{j=0}^{k} S(j) I(k-j)\right), \\
V(k+1) & =\frac{1}{k+1}\left((\lambda \theta) \delta(k)+\eta S(k)-\xi \beta \sum_{j=0}^{k} V(j) I(k-j)\right) \\
& -\frac{1}{k+1}(\zeta+\mu) V(k), \\
I(k+1) & =\frac{1}{k+1}\left(\beta \sum_{j=0}^{k} S(j) I(k-j)+\xi \beta \sum_{j=0}^{k} V(j) I(k-j)\right) \\
& -\frac{1}{k+1}(\tau+\varpi+\mu) I(k), \\
R(k+1) & =\frac{1}{k+1}(\varpi I(k)-\mu R(k)) .
\end{aligned}
$$


Table 1. The mathematical operations of the DTM.

\begin{tabular}{|c|c|}
\hline Original functions & Transformed functions \\
\hline$p(t)=m(t) \pm n(t)$ & $P(k)=M(k) \pm N(k)$ \\
$p(t)=c m(t)$ & $P(k)=c M(k)$, where $c$ is constant \\
$p(t)=1$ & $P(k)=\delta(k)$, where $\delta$ is Kronecker delta \\
$p(t)=\frac{d m(t)}{d t}$ & $P(k)=(k+1) M(k+1)$ \\
$p(t)=m(t) n(t)$ & $P(k)=\sum_{r}^{k}=0 M(r) N(k-r)$ \\
\hline
\end{tabular}

Therefore, the DTM series solution of the SVIR model in (1) is given as:

$$
\begin{aligned}
S(t) & =\sum_{k=0}^{n} S_{k} t^{k}, \\
V(t) & =\sum_{k=0}^{n} V_{k} t^{k}, \\
I(t) & =\sum_{k=0}^{n} I_{k} t^{k}, \\
R(t) & =\sum_{k=0}^{n} R_{k} t^{k} .
\end{aligned}
$$

The MSDTM solution of the SVIR rotavirus epidemic model in (1) is given below as:

$$
X(t)=\left\{\begin{array}{l}
\sum_{k=0}^{n} X_{1}(k) t^{k}, t \in\left[0, t_{1}\right] \\
\sum_{k=0}^{n} X_{2}(k)\left(t-t_{1}\right)^{k}, t \in\left[t_{1}, t_{2}\right] \\
\vdots \\
\sum_{k=0}^{n} X_{m}(k)\left(t-t_{M-1}\right)^{k}, \quad t \in\left[t_{M-1}, t_{M}\right]
\end{array}\right.
$$

where $X$ denotes $S, V, I$ or $R$ and $S_{i}, V_{i}, I_{i}$ and $R_{i}$ for $i=$ $1,2, \ldots, M$, to satisfy the recurrence relations given in (12):

$$
\begin{aligned}
S_{i}(k+1) & =\frac{1}{k+1}\left((\lambda-\lambda \theta) \delta(k)+\zeta V_{i}(k)-\eta S_{i}(k)\right) \\
& -\frac{1}{k+1}\left(\mu S_{i}(k)+\beta \sum_{j=0}^{k} S_{i}(j) I_{i}(k-j)\right) \\
V_{i}(k+1) & =\frac{1}{k+1}\left((\lambda \theta) \delta(k)-\xi \beta \sum_{j=0}^{k} V_{i}(j) I_{i}(k-j)\right) \\
& -\frac{1}{k+1}\left(-\eta S_{i}(k)+(\zeta+\mu) V_{i}(k)\right), \\
I_{i}(k+1) & =\frac{1}{k+1}\left(\beta \sum_{j=0}^{k} S_{i}(j) I_{i}(k-j)\right) \\
& \frac{1}{k+1}\left(+\xi \beta \sum_{j=0}^{k} V_{i}(j) I_{i}(k-j)\right) \\
& -\frac{1}{k+1}(\tau+\varpi+\mu) I_{i}(k), \\
R_{i}(k+1) & =\frac{1}{k+1}\left(\varpi I_{i}(k)-\mu R_{i}(k)\right),
\end{aligned}
$$

such that

$$
S_{i}(0)=S_{i-1}(0), \quad V_{i}(0)=V_{i-1}(0), \quad I_{i}(0)=I_{i-1}(0)
$$

and $R_{i}(0)=R_{i-1}(0)$.

\section{Result and Discussion}

The numerical experiments carried out using the DTM and MSDTM are presented in this section. It is noteworthy to point out that extensive analysis and numerical simulations on the epidemiological impact of the SVIR model used in this study have been carried out in the study of Omondi et al. (2015). Therefore, the numerical experiments carried out in this study only focus on two cases. Case 1 is to compare the solutions of the DTM and MSDTM with RK4 solutions using different decimal valued numbers as time t. Case 2 is to compare the solutions of the DTM and MSDTM with RK4 solutions on different days. All numerical experiments are carried using the free GNU Octave software (version 4.2.0), a free high-level programming language used for numerical computations and mostly compatible alternative to MATLAB [21]. For the numerical experiments, we consider (1) subject to the initial conditions given by

$$
S(0)=500, V(0)=350, I(0)=150, R(0)=50 .
$$

The list of parameter values used for the numerical simulation is provided in Table 2. Equations (9) and (13) and the parameter values given in Table 2 are used to perform all numerical experiments.

\subsection{Case 1}

In this subsection, decimal valued numbers are employed at time $t$ for the numerical experiment. The decimal values are randomly selected to compute the solutions of the DTM, MSDTM, and RK4 methods. The tables of solutions using the DTM, MSDTM, and RK4 for the susceptible, vaccinated, infected and removed compartments, respectively, at a different time $t$ are provided in Tables 3, 4, 5 and 6, respectively. Tables 3, 4, 5 and 6, respectively, show that DTM and MSDTM solutions $t=0.01,0.03,0.05,0.07,0.1,0.4,0.7$ and 1.0, respectively, converge to the solution of the RK4 method.

\subsection{Case 2}

In case 2, different numbers of days are selected for the numerical experiment. The tables of solutions using the DTM, MSDTM and RK4 for the susceptible, vaccinated, infected and removed compartments, respectively, at different days are provided in Tables 7, 8, 9 and 10, respectively. From Tables 7, 8, 9 and 10, respectively, when the DTM and MSDTM solutions are compared with the RK4 solutions at $t=$ 
Table 2. List of parameter values used for the numerical experiments.

\begin{tabular}{|c|c|c|c|}
\hline Parameter & Value & Unit & Reference \\
\hline$\lambda$ & 4.109 & per day & Assumed \\
$\theta$ & $1.884 \times 10^{-3}$ & per day & {$[15]$} \\
$\mu$ & $2.537 \times 10^{-5}$ & per day & {$[15]$} \\
$\tau$ & $4.466 \times 10^{-5}$ & per day & {$[15]$} \\
$\beta$ & $1.0 \times 10^{-4}$ & per day & Assumed \\
$\varpi$ & $9.9 \times 10^{-2}$ & per day & Assumed \\
$\xi$ & $1.0 \times 10^{-3}$ & per day & {$[15]$} \\
$\zeta$ & $2.778 \times 10^{-3}$ & per day & {$[15]$} \\
$\eta$ & $1.884 \times 10^{-3}$ & per day & {$[15]$} \\
\hline
\end{tabular}

Table 3. Solutions for $S(t)$ using DTM, MSDTM, and RK4 at $t=0.01,0.03,0.05,0.07,0.1,0.4,0.7$ and 1.0 , respectively.

\begin{tabular}{|c|c|c|c|}
\hline Time $(t)$ & DTM $S(t)$ & MSDTM $S(t)$ & RK4 $S(t)$ \\
\hline 0.01 & 499.9662102 & 499.9662294 & 499.9662100 \\
0.03 & 499.8987581 & 499.8988939 & 499.8987573 \\
0.05 & 499.8314754 & 499.7644599 & 499.8314741 \\
0.07 & 499.7643622 & 499.7644970 & 499.7643604 \\
0.1 & 499.6640094 & 499.6645950 & 499.6640069 \\
0.4 & 498.6812516 & 498.6812764 & 498.6812430 \\
0.7 & 497.7357472 & 497.7357925 & 497.7357329 \\
1.0 & 496.8267788 & 496.8268846 & 496.8267572 \\
\hline
\end{tabular}

Table 4. Solutions for $V(t)$ using DTM, MSDTM and RK4 at $t=0.01,0.03,0.05,0.07,0.1,0.4,0.7$ and 1.0 , respectively.

\begin{tabular}{|c|c|c|c|}
\hline Time $(t)$ & DTM $V(t)$ & MSDTM $V(t)$ & RK4 $V(t)$ \\
\hline 0.01 & 349.9996327 & 349.9996305 & 349.9996328 \\
0.03 & 349.9988963 & 349.9988875 & 349.9988967 \\
0.05 & 349.9981575 & 349.9981875 & 349.9981581 \\
0.07 & 349.9974163 & 349.9974272 & 349.9974172 \\
0.1 & 349.9963001 & 349.9963020 & 349.9963013 \\
0.4 & 349.9848485 & 349.9848422 & 349.9848526 \\
0.7 & 349.9728856 & 349.9728812 & 349.9728916 \\
1.0 & 349.9604321 & 349.9604216 & 349.9604394 \\
\hline
\end{tabular}

Table 5. Solutions for $I(t)$ using DTM, MSDTM and RK4 at $t=0.01,0.03,0.05,0.07,0.1,0.4,0.7$ and 1.0 , respectively.

\begin{tabular}{|c|c|c|c|}
\hline Time $(t)$ & DTM $I(t)$ & MSDTM $I(t)$ & RK4 $I(t)$ \\
\hline 0.01 & 149.9264625 & 149.9264632 & 149.9264630 \\
0.03 & 149.7794805 & 149.7794847 & 149.7794818 \\
0.05 & 149.6326224 & 149.6326328 & 149.6326246 \\
0.07 & 149.4858883 & 149.4859074 & 149.4858913 \\
0.1 & 149.2660194 & 149.2660565 & 149.2660236 \\
0.4 & 147.0826373 & 147.0104721 & 147.0826514 \\
0.7 & 144.9270054 & 144.9275948 & 144.9270244 \\
1.0 & 142.7989986 & 142.7990216 & 142.7990180 \\
\hline
\end{tabular}

Table 6. Solutions for $R(t)$ using DTM, MSDTM and RK4 at $t=0.01,0.03,0.05,0.07,0.1,0.4,0.7$ and 1.0 , respectively.

\begin{tabular}{|c|c|c|c|}
\hline Time $(t)$ & DTM $R(t)$ & MSDTM $R(t)$ & RK4 $R(t)$ \\
\hline 0.01 & 50.1484506 & 50.1484509 & 50.1484509 \\
0.03 & 50.4451333 & 50.4451342 & 50.4451342 \\
0.05 & 50.7415249 & 50.7415265 & 50.7415265 \\
0.07 & 51.0376257 & 51.0376281 & 51.0376280 \\
0.1 & 51.4812321 & 51.4812356 & 51.4812355 \\
0.4 & 55.8815180 & 55.8815433 & 55.8815359 \\
0.7 & 60.2173283 & 60.2174089 & 60.2173692 \\
1.0 & 64.4894829 & 64.4896713 & 64.4895582 \\
\hline
\end{tabular}

$10,20,30,40,50,60,70,80,90$ and 100, respectively, for all compartments, only the solutions of the MSDTM method converge to the RK4 solutions. The DTM solutions of the susceptible compartment converge with RK4 at days 10, 20 and 30 , respectively. However, the reverse is the case from 40 days to 100 days. In the vaccinated compartment, DTM solutions converge to RK4 from 10 to 70 days. On the other hand, the
DTM solutions do not converge to the RK4 solutions from 80 to 100 days. Similarly, in the infected compartment, the DTM solutions converge to the solutions of the RK4 method from 10 to 50 days and diverge from 60 to 100 days. In the removed compartment, the solutions of the DTM converge to the RK4 solutions on 10, 20 and 30 days, respectively, but this diverges from 40 to 100 days. As stated earlier in the "Introduc- 
Table 7. Solutions for $S(t)$ using DTM, MSDTM, and RK4 at $t=10,20,30,40,50,60,70,80,90$ and 100, respectively.

\begin{tabular}{|c|c|c|c|}
\hline Days & DTM $S(t)$ & MSDTM $S(t)$ & RK4 $S(t)$ \\
\hline 10 & 483.5297444 & 483.5192425 & 483.5177420 \\
20 & 490.4456618 & 490.3558445 & 490.3567408 \\
30 & 506.9798678 & 509.9446202 & 509.9467134 \\
40 & 528.4542784 - - Divergence & 536.6945051 & 536.6955034 \\
50 & 538.4996875 - - Divergence & 567.5109384 & 567.5155369 \\
60 & 525.4344224 - - Divergence & 600.6165418 & 600.6328543 \\
70 & 477.6929971 - - Divergence & 634.9615953 & 635.0080919 \\
80 & 392.371418 - - Divergence & 669.9114025 & 670.0271358 \\
90 & 285.7245544 - - Divergence & 705.0764109 & 705.2570114 \\
100 & 207.1900000 - - Divergence & 740.2136041 & 740.3932005 \\
\hline
\end{tabular}

Table 8. Solutions for $V(t)$ using DTM, MSDTM, and RK4 at $t=10,20,30,40,50,60,70,80,90$ and 100, respectively.

\begin{tabular}{|c|c|c|c|}
\hline Days & DTM $V(t)$ & MSDTM $V(t)$ & RK4 $V(t)$ \\
\hline 10 & 349.4476311 & 349.4473511 & 349.4473551 \\
20 & 348.8620227 & 348.8597274 & 348.8597347 \\
30 & 348.5843628 & 348.5555195 & 348.5555235 \\
40 & 348.6248051 & 348.7024677 & 348.7024667 \\
50 & 349.0462500 & 349.3871520 & 349.3873360 \\
60 & 349.6243059 & 350.6508146 & 350.6518110 \\
70 & 350.1396426 & 352.5087307 & 352.5121506 \\
80 & 350.6159693 - - Divergence & 354.9605791 & 354.9707281 \\
90 & 351.8470546 - - Divergence & 357.9967874 & 358.0151918 \\
100 & 356.2600000 - - Divergence & 361.6027180 & 361.6240369 \\
\hline
\end{tabular}

Table 9. Solutions for $I(t)$ using DTM, MSDTM and RK4 at $t=10,20,30,40,50,60,70,80,90$ and 100, respectively.

\begin{tabular}{|c|c|c|c|}
\hline Days & DTM $I(t)$ & MSDTM $I(t)$ & RK4 $I(t)$ \\
\hline 10 & 90.8739924 & 90.8718208 & 90.8703508 \\
20 & 54.8825832 & 54.8519417 & 54.8498916 \\
30 & 32.8629060 & 33.5708323 & 33.5703129 \\
40 & 18.3039680 & 21.0349321 & 21.0347201 \\
50 & 14.9350000 & 13.5676162 & 13.5671466 \\
60 & -670.1056800 - Divergence & 9.0363898 & 9.0354898 \\
70 & $-1,304.184830$ - - Divergence & 6.2253380 & 6.2237110 \\
80 & $-2,297.567680$ - - Divergence & 4.4401424 & 4.4373823 \\
90 & $-3,764.955630$ - - Divergence & 3.2799574 & 3.2768044 \\
100 & $-5,835.830000$ - - Divergence & 2.5096538 & 2.5074879 \\
\hline
\end{tabular}

Table 10. Solutions for $R(t)$ using DTM, MSDTM and RK4 at $t=10,20,30,40,50,60,70,80,90$ and 100, respectively.

\begin{tabular}{|c|c|c|c|}
\hline Days & DTM $R(t)$ & MSDTM $R(t)$ & RK4 $R(t)$ \\
\hline 10 & 166.9071569 & 166.9274854 & 166.9302349 \\
20 & 237.3142082 & 237.4746808 & 237.4755688 \\
30 & 278.9018682 & 280.2494560 & 280.2478163 \\
40 & 287.4426068 - Divergence & 306.66391546 & 306.6631358 \\
50 & 317.0357813 - - Divergence & 323.3997866 & 323.3952880 \\
60 & 347.0983714 - - Divergence & 334.3233127 & 334.3069161 \\
70 & 671.3517800 - - Divergence & 341.6846690 & 341.6361560 \\
80 & 865.4108800 - - Divergence & 346.8118904 & 346.6885902 \\
90 & $1,108.810580$ - - Divergence & 350.5047657 & 350.3088658 \\
100 & $1,399.670000$ - - Divergence & 353.2562937 & 353.0572452 \\
\hline
\end{tabular}

tion" section, DTM provides an excellent solution in a small region, which is evident in the solutions obtained in case 1 of this study. However, the same convergence exhibited in case 1 is not found in case 2 when larger values are selected for the numerical experiments.

\subsection{Graphical plot of DTM, MSDTM, and RK4 solutions}

In this subsection, the graphical illustration of solutions obtained from the DTM, MSDTM and RK4 are presented. The DTM, MSDTM, and RK4 solutions in Tables 7, 8, 9 and 10 are employed to generate the plots. Figures 1, 2, 3 and 4, respec- tively, denote the graphical representation of the DTM, MSDTM and RK4 solutions obtained from the susceptible, vaccinated, infected, and removed compartments.

From Figures 1, 2, 3 and 4, respectively, the solutions of MSDTM are in good agreement with the solutions of the RK4 method. The absolute error solutions indicates that the MSDTM has a small error value when it is compared to the RK4 while DTM has a large error value. The absolute error computations further proves that the MSDTM has a better computation efficiency than the DTM. 
Table 11. The absolute error computed from the DTM and MSDTM along with the result obtained by the RK4 method.

\begin{tabular}{|c|c|c|c|c|}
\hline Days & DTM for $S(t)$ & MSDTM for $S(t)$ & -DTM-RK4- & -MSDTM-RK4- \\
\hline 10 & 483.529744 & 483.5192425 & 0.0120020 & 0.0015005 \\
\hline 20 & 490.4456618 & 490.3558445 & 0.0889210 & 0.0008963 \\
\hline 30 & 506.9798678 & 509.9446202 & 2.9668456 & 0.0020932 \\
\hline 40 & 528.4542784 & 536.6945051 & 8.2412250 & 0.0009983 \\
\hline 50 & 538.4996875 & 567.5109384 & 29.0158494 & 0.0045985 \\
\hline 60 & 525.4344224 & 600.6165418 & 75.1984319 & 0.0163125 \\
\hline 70 & 477.6929971 & 634.9615953 & 157.3150948 & 0.0464966 \\
\hline 80 & 392.3714180 & 669.9114025 & 277.6557178 & 0.1157333 \\
\hline 90 & 285.7245544 & 705.0764109 & 419.5324570 & 0.1806005 \\
\hline 100 & 207.1900000 & 740.2136041 & 533.2032005 & 0.1795964 \\
\hline Days & DTM for $V(t)$ & MSDTM for $V(t)$ & -DTM-RK4- & -MSDTM-RK4- \\
\hline 10 & 349.4476311 & 349.4473511 & 0.0002760 & 0.0000040 \\
\hline 20 & 348.8620227 & 348.8597274 & 0.0022880 & 0.0000073 \\
\hline 30 & 348.5843628 & 348.5555195 & 0.0288393 & 0.0000040 \\
\hline 40 & 348.6248051 & 348.7024677 & 0.0776616 & 0.0000010 \\
\hline 50 & 349.0462500 & 349.3871520 & 0.3410860 & 0.0001840 \\
\hline 60 & 349.6243059 & 350.6508146 & 1.0275051 & 0.0009964 \\
\hline 70 & 350.1396426 & 352.5087307 & 2.3725080 & 0.0034199 \\
\hline 80 & 350.6159693 & 354.9605791 & 4.3547588 & 0.0101490 \\
\hline 90 & 351.8470546 & 357.9967874 & 6.1681372 & 0.0184044 \\
\hline 100 & 356.2600000 & 361.6027180 & 5.3640369 & 0.0213189 \\
\hline Days & DTM for $I(t)$ & MSDTM for $I(t)$ & -DTM-RK4- & -MSDTM-RK4- \\
\hline 10 & 90.8739924 & 90.8718208 & 0.0036416 & 0.0014700 \\
\hline 20 & 54.8825832 & 54.8519417 & 0.0326916 & 0.0020501 \\
\hline 30 & 32.8629060 & 33.5708323 & 0.7074069 & 0.0005194 \\
\hline 40 & 18.3039680 & 21.0349321 & 2.7307521 & 0.0002120 \\
\hline 50 & 14.9350000 & 13.5676162 & 1.3678534 & 0.0004696 \\
\hline 60 & -670.1056800 & 9.0363898 & 661.0701902 & 0.0009000 \\
\hline 70 & $-1,304.184830$ & 6.2253380 & 1297.9611190 & 0.0016270 \\
\hline 80 & $-2,297.567680$ & 4.4401424 & 2293.1302977 & 0.0027601 \\
\hline 90 & $-3,764.955630$ & 3.2799574 & 3761.6788256 & 0.0031526 \\
\hline 100 & $-5,835.830000$ & 2.5096538 & 5833.3225121 & 0.0021651 \\
\hline Days & DTM for $R(t)$ & MSDTM for $R(t)$ & -DTM-RK4- & -MSDTM-RK4- \\
\hline 10 & 166.9071569 & 166.9274854 & 0.0230780 & 0.0027495 \\
\hline 20 & 237.3142082 & 237.4746808 & 0.1613606 & 0.0008880 \\
\hline 30 & 278.9018682 & 280.2494560 & 1.3459481 & 0.0016397 \\
\hline 40 & 287.4426068 & 306.6639155 & 19.2205290 & 0.0007797 \\
\hline 50 & 317.0357813 & 323.3997866 & 6.3595067 & 0.0044986 \\
\hline 60 & 347.0983714 & 334.3233127 & 12.7914553 & 0.0163966 \\
\hline 70 & 671.3517800 & 341.6846690 & 329.7156240 & 0.0485130 \\
\hline 80 & 865.4108800 & 346.8118904 & 518.7222898 & 0.1233002 \\
\hline 90 & $1,108.810580$ & 350.5047657 & 758.5017142 & 0.1958999 \\
\hline 100 & $1,399.670000$ & 353.2562937 & 1046.6127548 & 0.1990485 \\
\hline
\end{tabular}

\subsection{Convergence of the proposed methods and their ab- solute errors}

In this subsection, the convergence of the DTM and MSDTM solutions against the RK4 solutions are discussed. The RK4 is an effective and widely used method that offers a good balance between the order of accuracy and computation cost. Hence, this is the reason for comparing the DTM and MSDTM solutions with the RK4 solutions. As shown in Tables 3, 4, 5 and 6, respectively, the DTM and MSDTM solutions converge to the RK4 solutions when the time interval $\mathrm{t}$ is small. However, in Tables 7, 8, 9 and 10, all DTM solutions do not converge to the RK4 solutions at the different time $t$ values. The DTM solutions diverge in the susceptible compartment from 40 days to 100 days, the vaccinated compartment from 80 to 100 days, the infected compartment from 60 to 100 days, and the recovered compartment from 40 to 100 days. Thus, from the tabular solutions and graphical illustrations presented in this study, the MSDTM has a small and large convergence interval. In contrast, the DTM has only a small interval of convergence.
The absolute errors of DTM and MSDTM solutions are presented in Table 11.

\section{Conclusions}

In this study, we apply the MSDTM method to solve the SVIR rotavirus epidemic model with given initial conditions. To examine the accuracy of the MSDTM, its solutions are compared with the solutions of the SVIR model using the RK4 method. Numerical calculations are performed at different time intervals and days to examine the efficiency of the DTM and MSDTM. The numerical experiments showed that when decimal valued numbers are used for the numerical experiments, the DTM and MSDTM converge to the RK4 solutions of the SVIR model. However, when the large valued numbers are used for the numerical experiments, the MSDTM converges well to the RK4 method than the DTM. As stated in a recent study by Shahrear et al. (2018) [22] the DTM is only effective for the approximation of the solutions when a minimal time 


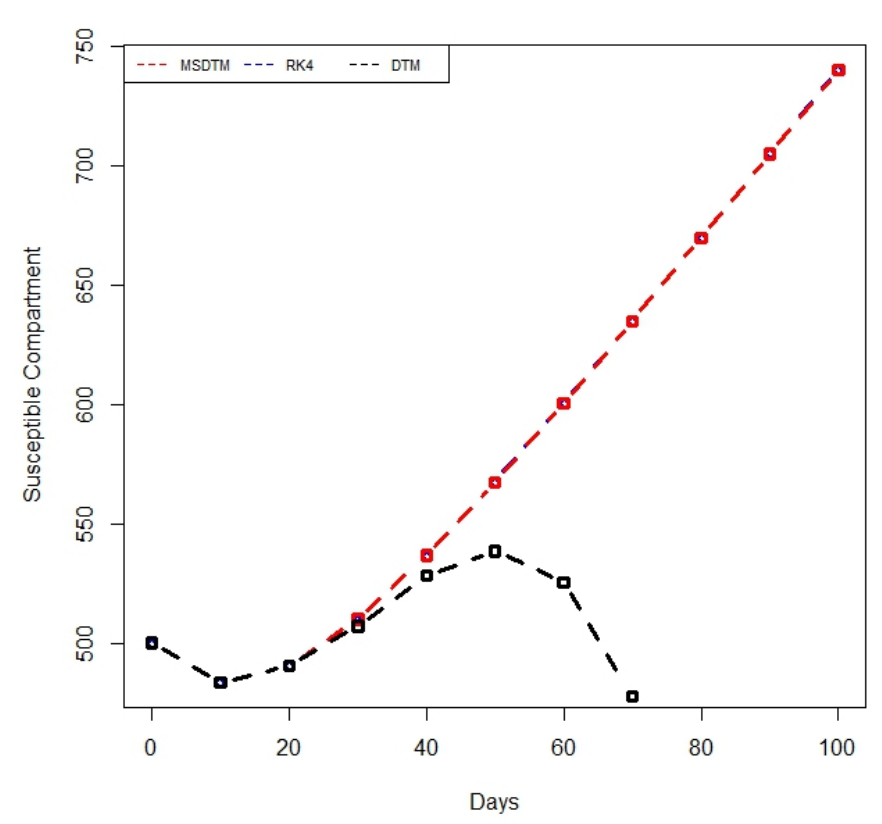

Figure 1. The comparison of the DTM, MSDTM and RK4 solutions for the susceptible compartment at $t=10,20,30,40,50,60,70,80,90$ and 100, respectively.

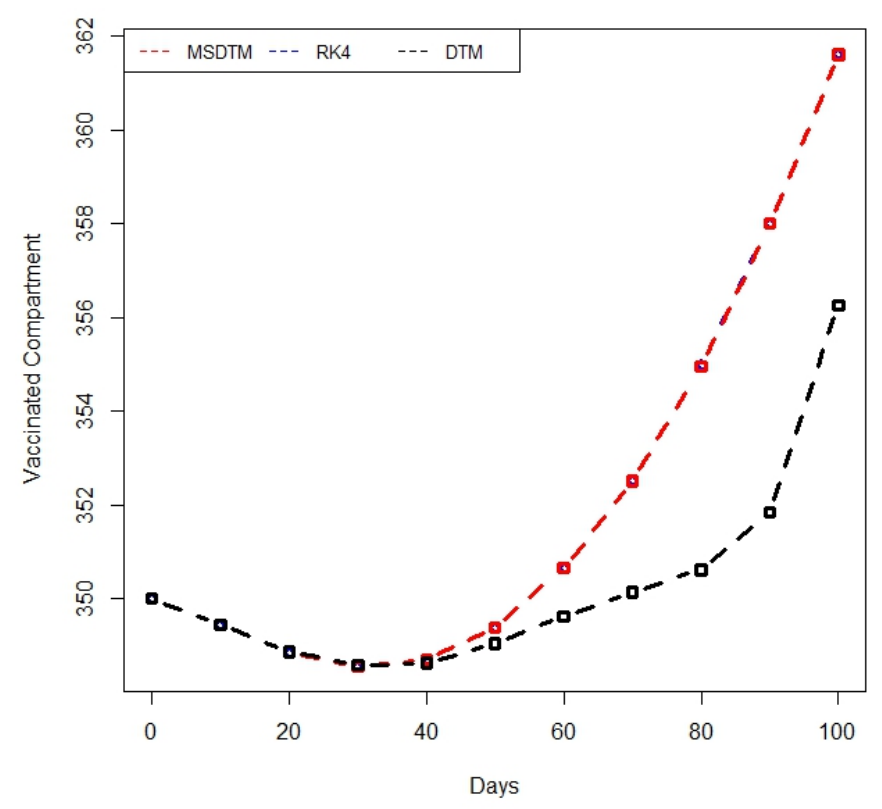

Figure 2. The comparison of the DTM, MSDTM and RK4 solutions for the vaccinated compartment at $t=10,20,30,40,50,60,70,80,90$ and 100, respectively.

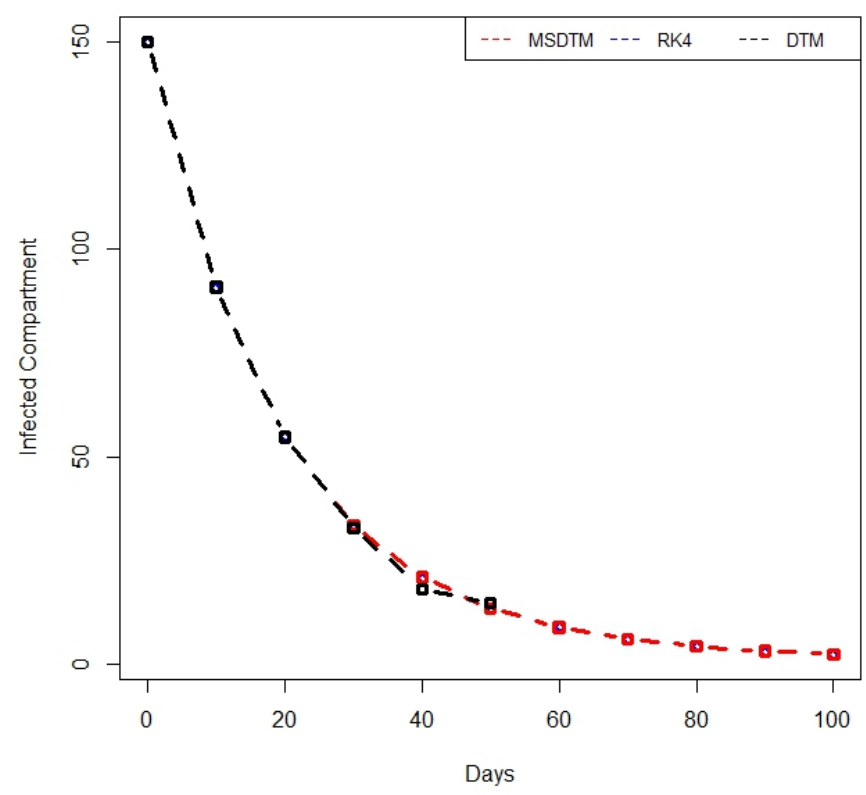

Figure 3. The comparison of the DTM, MSDTM and RK4 solutions for the infected compartment at $t=10,20,30,40,50,60,70,80,90$ and 100 , respectively.

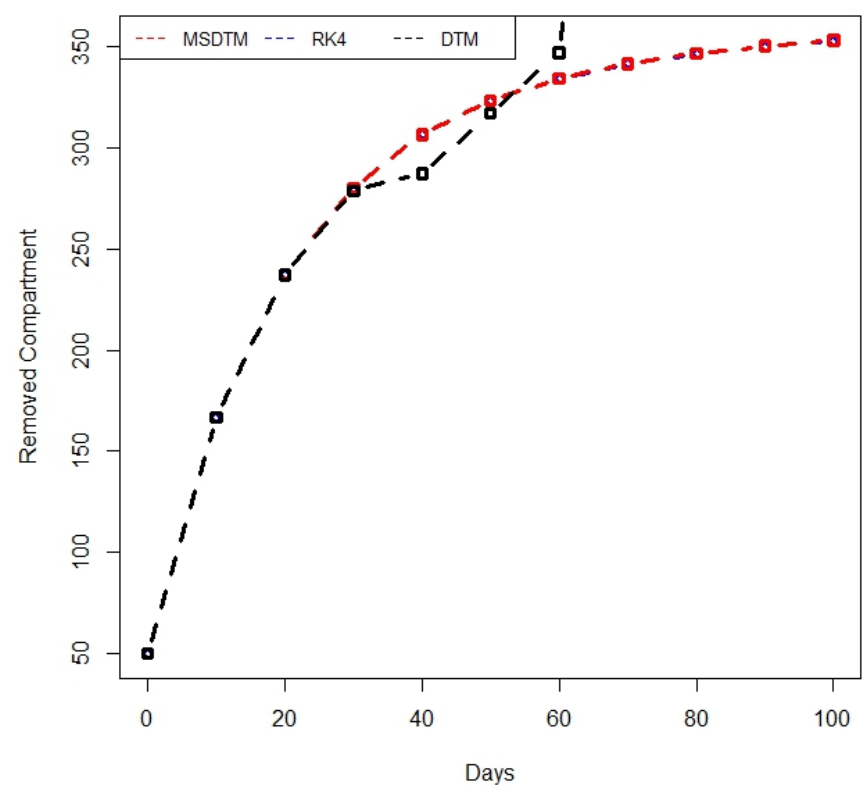

Figure 4. The comparison of the DTM, MSDTM and RK4 solutions for the removed compartment at $t=10,20,30,40,50,60,70,80,90$ and 100 , respectively. 
frame is considered. The result found in the study of [22] is similar to the obtained DTM results in the current study. Thus, we advise future researchers only to consider the application of DTM for solving problems with small-time intervals. Furthermore, we suggest the application of the MSDTM to other epidemic models of various compartmental sizes to ascertain its efficiency.

\section{Acknowledgements}

The author would like to thank the editors and the anonymous reviewers for their constructive and helpful comments.

\section{REFERENCES}

[1] Heslop D. J., Chughtai A. A., "Publicly available software tools for decision-makers during an emergent epidemic-Systematic evaluation of utility and usability," Epidemics, vol. 21, pp. 1-12, 2017.

[2] Towers S., "Introduction to compartmental modelling, Polymatheia," Archived from the original on December 11, 2013, Retrieved December 11, 2013.

[3] Brauer F., "Compartmental models in epidemiology," Silver Burdett Press, Londyn, 1986.

[4] Saddiq S. F., Khan M. A., Khan S. A., Ahmad F., Ullah M., "Analytical solution of an SEIV epidemic model by homotopy perturbation method," VFAST Transactions on Mathematics, vol. 1, pp. 1-7, 2013.

[5] Wazwaz A. M., "A comparison between Adomian decomposition method and Taylor series method in the series solutions," Applied Mathematics and Computation, vol. 97, pp. 37-44, 1998.

[6] He J. H., "Comparison of homotopy perturbation method and homotopy analysis method," Applied Mathematics and Computation, vol. 156, pp. 527-539, 2004.

[7] He J. H., "Homotopy perturbation technique," Computer methods in applied mechanics and engineering, vol. 178, pp. 257-262, 1999.

[8] He J. H., "An elementary introduction to the homotopy perturbation method," Computers and Mathematics with Applications, vol. 57, pp. 410-412, 2009.

[9] Ndii M. Z., Anggriani N., Supriatna A. K., "Application of differential transformation method for solving dengue transmission mathematical model," In AIP conference proceedings (vol. 1937, no. 1, pp. 020012) (2018, March). AIP Publishing LLC.
[10] Peter O., Ibrahim M. O., Oguntolu F. A., Akinduko O. B., Akinyemi S. T., "Direct and indirect transmission dynamics of typhoid fever model by differential transform method," ATBU, Journal of Science, Technology and Education (JOSTE), vol. 6, no. 1, pp. 167-177, 2018.

[11] Kharrat B. N., Toma G., "Differential transform method for solving initial and boundary value problems represented by linear and nonlinear ordinary differential equations of 14 Order th," World Applied Sciences Journal, vol. 37, no. 6, pp. 481-485, 2019.

[12] Christopher A. J., Magesh N., Preethi G. T., "Dynamical analysis of corona-virus (COVID-19) epidemic model by differential transform method," Research Square (preprint), 2020. DOI: $10.21203 /$ rs.3.rs-25819/v1

[13] Jang M. J., Chen C. L., Liy Y. C., "On solving the initial-value problems using the differential transformation method," Applied Mathematics and Computation, vol. 115, pp. 145-160, 2000 .

[14] Bervillier C., "Status of the differential transformation method," Applied Mathematics and Computation, vol. 218, pp. 1015810170, 2012.

[15] Gökdoğan A., Merdan M., Yildirim A., "Adaptive multi-step differential transformation method to solving nonlinear differential equations," Mathematical and Computer Modelling, vol. 55, pp. 761-769, 2012.

[16] Gökdoğan A., Merdan M., Yildirim A., "A multistage differential transformation method for approximate solution of Hantavirus infection model," Mathematical and Computer Modelling, vol. 55, pp. 761-769, 2012.

[17] Astuti F., Suryanto A., Darti I., "Multi-step differential transform method for solving the influenza virus model with disease resistance," 9th Annual Basic Science International Conference (BaSIC2019) Malang, Indonesia, 20-21 March 2019, 05201, 2019.

[18] Omondi O. L., Wang C., Xue X., Lawi O. G., "Modeling the effects of vaccination on rotavirus infection," Advances in Difference Equations, vol. 381, pp. 1-12, 2015.

[19] Hassan I. A. H., "Differential transformation technique for solving higher-order initial value problems," Applied Mathematics and Computation, vol. 154, pp. 299-311, 2004.

[20] Batiha K., Batiha B., "A new algorithm for solving linear ordinary differential equations," World Applied Sciences Journal, vol. 15, pp. 1774-1779, 2004.

[21] Eaton J. W., Bateman D., Hauberg S., Wehbring R., "GNU Octave version 4.2.0 manual: a high level interactive language for numerical computations," 2016. 
[22] Shahrear P., Chakraborty A. K., Islam, M. A., Habiba U., "Analysis of computer virus propagation based on compartmental model," Applied Mathematics and Computation, vol.
97, pp. 33-44, 2018. 